\title{
Two-Layers CE Algorithm for Flexible Job Shop Scheduling Problem with Electric Power Cost Considered
}

\author{
G.R. WANG
}

School of Control Science and Engineering, Shandong University, Jinan, Shandong, China

Key Laboratory of Building Renewable Energy Utilization Technologies, Ministry of Education (Shandong Jianzhu University), Jinan, Shandong, China

Q.Q. LI*

School of Control Science and Engineering, Shandong University, Jinan, Shandong, China

\begin{abstract}
Propose a Two-Layers CE algorithm to solve flexible job shop scheduling problem. Two optimal objectives are considered: makespan and electric power cost. Analyze the influence of different values of the weight vector to the two objectives. Different simulation instances with different problem scale are devised to validate the superior performance of the algorithm.
\end{abstract}

KEYWORD: Cross entropy; FJSP; Scheduling; Electric power cost

\section{INTRODUCTIOS}

Flexible job shop scheduling problem (FJSP) is the extension of the traditional job shop scheduling problem (JSP). The machine selection of FJSP is more flexible and more suitable for the actual production. But it is more complicated and difficult to solve than JSP, therefore it becomes a hot topic for research.

Recently, energy crisis is becoming more serious. Reducing energy costs becomes another important objective for industrial enterprises. Power consumption accounts for a large part in total energy consumption in the field of machine processing. In the environment of time-of-use electricity price (TOU), if the electric power cost (EPC) is viewed as an objective then expensive electricity will be avoided. So, in this study, minimizing the MS and minimizing the EPC are all considered, and a weight vector is given for the decision maker to selecting the different preference to this two objectives.

FJSP is NP-hard and more complicated than traditional JSP. No polynomial time algorithm can solve it accurately (Blazewicz et al. 1988). Now, the methods widely used are heuristic rules (Blazewicz et al. 1996, Benziani et al. 2014) and intelligent search algorithms such as GA and so on (Pezzella et al. 2005, Zhang et al. 2011). The cross entropy (CE) method is a recent generic Monte Carlo technique for solving complicated simulation and optimization problems (De Boer et al. 2005). It has been applied in many areas. In this paper, a Two-Layers CE algorithm is proposed to solve the FJSP with two probability models built to generate the machine assignment status and operation sequence seperately. Details can be found in section 3 .

\section{PROBLEM DESCRIPTION}

FJSP is defined that there are $n$ jobs will be processed on $m$ machines. Each job has one or more operations and each operation can be processed on one of the allowed machines. Process time is different on different machine. Schedule objective is to select a suitable machine for an operation and determine its start time on the machine, and make some required property indicators optimal. It includes two subproblems: determine the machine for each operation and determine the sequence of operations on each machine (Gao et al. 2012). Constraint conditions of the FJSP are as follows.

(1) Only one operation can be processed on a machine at the same time.

(2) Only one machine can process an operation of a job.

(3) The processing of an operation can not be interrupted.

(4) The operations of a job have precedence relation, but it doesn't exist between different jobs.

(5) All jobs can be processed at time zero.

In this paper, two optimal objectives are considered. One is to minimize the MS, the other is to minimize the EPC. In calculating the EPC, we also consider the power consumption when the machine is idle. This is beneficial to improve 
productivity by guarantying little idle time of a machine after its startup.

\section{RESOLVING METHOD}

\subsection{The CE method}

The CE method is based on Markov method and an important sampling technology, and through statistical learning from the elite samples and updating the probability model it can find the optimal or near-optimal solution. It was introduced by Rubinstein (1999) for rare-event probability estimation. For the minimum problem, the $\mathrm{CE}$ method is to connect solving $S\left(x^{*}\right)=\gamma^{*}=\min S(x)$ in a feasible region $\chi$ with estimating probability of the event $\{S(x) \leq \gamma\}$, that is, $\ell=P_{u}(S(X) \leq \gamma)$ $=\sum_{x} I_{\{S(x) \leq \gamma\}} f(x, u)=E_{u} I_{\{S(x) \leq \gamma\}}$, where $x$ has some probability density $f(x, u)$ on $\chi$ and $\gamma$ is some level. $I_{\{S(x) \leq y\}}$ is indicator function and $I_{\{S(x) \leq \gamma\}}=\left\{\begin{array}{ll}1, & S(x) \leq \gamma \\ 0, & \text { others }\end{array}\right.$.

The CE method is a multilevel updating algorithm. By constructing a distribution parameter sequence $\left\{v_{t}, t \geq 0\right\}$ and a level sequence $\left\{\gamma_{t}, t \geq 0\right\}$, through multilevel iterations, it generates a series of $\left\{\gamma_{1}, v_{1}\right\}$ starting from $\left\{\gamma_{1}, v_{1}\right\}$. After $T$ times iterations, $\left\{\gamma_{T}, v_{T}\right\}$ is very close to the optimal $\left\{\gamma^{*}, v^{*}\right\}$, then $\gamma_{T}$ is regarded as the minimum value of $S$. More details can be found on the CE method homepage: www.cemethod.org. Based on the problem description in section 3, we proposed a Two-Layers $\mathrm{CE}$ algorithm to solve the FJSP.

\subsection{Encoding, decoding and probability updating mechanism}

\subsubsection{Encoding}

A matrix coding scheme is proposed in the TwoLayers CE algorithm. Matrix $M \boldsymbol{A}_{n \times m}$ and $O S_{n \times n}$ represents separately the machine assignment status and the operation sequence, where $n$ represents the number of total operations, $m$ represents the number of machines. The element $m a_{i j}$ of matrix $M A_{n \times m}$ represents whether or not operation $i$ is assigned to machine $j$ (where $i=\sum_{k=1}^{(p-1)} o n_{k}+q$, $o n_{k}$ indicates the number of operations of job $k, q$ indicates the $q_{t h}$ operations of job $p$ ). The element $o s_{i j}$ of matrix $O S_{n \times n}$ represents whether or not operation $i$ is in the position $j$ of the sequence of all operations. $m a_{i j}$ and $o s_{i j}$ conform to Equations 1 and 2.

$$
\begin{aligned}
& \sum_{j=1}^{m} m a_{i j}=1, i=1,2, \cdots, n, m a_{i j} \in\{0,1\} \\
& \sum_{j=1}^{n} o s_{i j}=1, i=1,2, \cdots, n, o s_{i j} \in\{0,1\}
\end{aligned}
$$

\subsubsection{Decoding}

Based on the encoding matrix $M A_{n \times m}$ and $O S_{n \times n} a$, the decoding method will give an integrated solution of the FJSP expressed as the matrix $\boldsymbol{S} \boldsymbol{T}_{n \times m}$. The element $s t_{i j}$ represents the start time of operation $i$ on machine $j$. If operation $i$ cannot be processed by machine $j, s t_{i j}$ is set to be a large constant. The decoding method we adopted is an operation-plug-in method which is proposed by Gao et al. 2012.

\subsubsection{Initialization and updating mechanism of the probability model}

In the Two-Layers CE algorithm for FJSP, the probability model is defined separately as $\boldsymbol{P}_{n \times m}$ and $Q_{n \times n}$. The element $p_{i j}$ of matrix $\boldsymbol{P}_{n \times m}$ represents the probability of operation $i$ is assigned to machine $j$. The element $q_{i j}$ of matrix $Q_{n \times n}$ represents the probability of operation $i$ is in the position $j$ of the sequence of all operations. $p_{i j}$ and $q_{i j}$ conform to Equations 3 and 4.

$$
\begin{gathered}
\sum_{j=1}^{m} p_{i j}=1, i=1,2, \cdots, n, p_{i j} \in[0,1] \\
\sum_{j=1}^{n} q_{i j}=1, i=1,2, \cdots, n, q_{i j} \in[0,1]
\end{gathered}
$$

In information theory, the information entropy of the generated data according to a uniform probability distribution is maximal. So we use a uniform probability distribution to initialize the probability model. That is, $p_{i j}^{0}=1 / m, q_{i j}^{0}=1 / n$.

In particular, because not all machines can be selected to process an operation, some elements in $\boldsymbol{P}_{n \times m}$ should always be set to zeros. Consequently the initial probability model $P^{0}$ will be modified as $p_{i j}^{0}=1 / m_{i}$, where $m_{i}$ is the number of machines can be selected to process operation $i$ and if operation $i$ cannot be processed by machine $j$ then $p_{i j}^{0}=0$. Similarly, because the sequence of operations of a 
job is known in advance, an operation can not be in some positions, then some elements in $Q_{n \times n}$ should always be set to zeros. Consequently the initial probability model $Q^{0}$ will be modified as $q_{i j}^{0}=1 /\left(n-\left(o n_{k}-1\right)\right)$, here, operation $i$ belongs to job $k$. Meanwhile, if $o n_{k} \geq 2$ and operation $i$ is the first operation of job $k$ then it cannot be in the position of the last one, that is $q_{i n}^{0}=0$ and so on. Take the partial FJSP (P-FJSP) given in Table 1 as example, the modification process is shown in Figure 1.

Table 1. Example of P-FJSP.

\begin{tabular}{|c|c|c|c|c|c|}
\hline \multirow{2}{*}{ Job } & \multirow{2}{*}{ Operation } & \multicolumn{4}{|c|}{ Machine } \\
\cline { 2 - 6 } 1 & $\mathrm{M} 1$ & $\mathrm{M} 2$ & $\mathrm{M} 3$ & $\mathrm{M} 4$ \\
\hline \multirow{3}{*}{1} & $1\left(^{O_{11}}\right)$ & 2 & 5 & 4 & 3 \\
\hline \multirow{3}{*}{2} & $2\left(\left(_{12}\right)\right.$ & -- & 6 & -- & 4 \\
\cline { 2 - 6 } & $3\left(\left(_{21}\right)\right.$ & 7 & 5 & 8 & -- \\
\cline { 2 - 6 } & $4\left({ }^{O_{22}}\right)$ & -- & 4 & 6 & 3 \\
\cline { 2 - 6 } & $5\left({ }^{O_{23}}\right)$ & 8 & -- & 7 & -- \\
\hline
\end{tabular}

Table 2. Simulation results.

\begin{tabular}{|c|c|c|c|c|}
\hline \multicolumn{2}{|c|}{$w=\left[w_{m s}, w_{\text {epc }}\right]$} & {$[1,0]$} & {$[0.6,0.4]$} & {$[0,1]$} \\
\hline \multirow{2}{*}{ Instance1 } & MS (h) & 11 & 11.3 & 11.3 \\
\cline { 2 - 5 } & EPC (yuan) & 24.08 & 22.17 & 21.73 \\
\hline \multirow{2}{*}{ Instance2 } & MS (h) & 17 & 18.2 & 20.3 \\
\cline { 2 - 5 } & EPC (yuan) & 102.13 & 85.27 & 82.36 \\
\hline \multirow{2}{*}{ Instance3 } & MS (h) & 20.45 & 21.6 & 23.2 \\
\cline { 2 - 5 } & EPC (yuan) & 163.047 & 139.85 & 123.998 \\
\hline
\end{tabular}

The updating process of probability distribution is the critical step of the Two-Layers CE algorithm. In this paper, we adopt the updating mechanism described as Equations 5 and 6.

$$
\begin{aligned}
& p_{i j}^{k}=\left(1-\alpha_{1}\right) \cdot p_{i j}^{k-1}+\alpha_{1} \cdot \frac{1}{\rho_{1} \cdot N_{1}} \sum_{l=1}^{\rho_{1} \cdot N_{1}} I_{\left\{m a_{i j}=1\right\}}^{l} \\
& q_{i j}^{k}=\left(1-\alpha_{2}\right) \cdot q_{i j}^{k-1}+\alpha_{2} \cdot \frac{1}{\rho_{2} \cdot N_{2}} \sum_{l=1}^{\rho_{2} \cdot N_{2}} I_{\left\{s_{i j}=1\right\}}^{l}
\end{aligned}
$$

Here, $\alpha_{1}, \alpha_{2} \in(0,1)$ are the learning rates, $I_{\left\{m a_{i j}=1\right\}}^{l}$ and $I_{\left\{o s_{i j}=1\right\}}^{l}$ are indicator functions and $\rho_{1} \cdot N_{1}, \rho_{2} \cdot N_{2}$ are the numbers of elite samples.

$$
\begin{aligned}
P^{0}= & {\left[\begin{array}{cccc}
\frac{1}{4} & \frac{1}{4} & \frac{1}{4} & \frac{1}{4} \\
\frac{1}{4} & \frac{1}{4} & \frac{1}{4} & \frac{1}{4} \\
\frac{1}{4} & \frac{1}{4} & \frac{1}{4} & \frac{1}{4} \\
\frac{1}{4} & \frac{1}{4} & \frac{1}{4} & \frac{1}{4} \\
\frac{1}{4} & \frac{1}{4} & \frac{1}{4} & \frac{1}{4}
\end{array}\right] \stackrel{\text { Modify }}{\Rightarrow} P^{0}=\left[\begin{array}{cccc}
\frac{1}{4} & \frac{1}{4} & \frac{1}{4} & \frac{1}{4} \\
0 & \frac{1}{2} & 0 & \frac{1}{2} \\
\frac{1}{3} & \frac{1}{3} & \frac{1}{3} & 0 \\
0 & \frac{1}{3} & \frac{1}{3} & \frac{1}{3} \\
\frac{1}{2} & 0 & \frac{1}{2} & 0
\end{array}\right] } \\
Q^{0} & =\left[\begin{array}{ccccc}
\frac{1}{5} & \frac{1}{5} & \frac{1}{5} & \frac{1}{5} & \frac{1}{5} \\
\frac{1}{5} & \frac{1}{5} & \frac{1}{5} & \frac{1}{5} & \frac{1}{5} \\
\frac{1}{5} & \frac{1}{5} & \frac{1}{5} & \frac{1}{5} & \frac{1}{5} \\
\frac{1}{5} & \frac{1}{5} & \frac{1}{5} & \frac{1}{5} & \frac{1}{5} \\
\frac{1}{5} & \frac{1}{5} & \frac{1}{5} & \frac{1}{5} & \frac{1}{5}
\end{array}\right] \stackrel{\text { Modify }}{\Rightarrow} Q^{0}=\left[\begin{array}{ccccc}
\frac{1}{4} & \frac{1}{4} & \frac{1}{4} & \frac{1}{4} & 0 \\
0 & \frac{1}{4} & \frac{1}{4} & \frac{1}{4} & \frac{1}{4} \\
\frac{1}{3} & \frac{1}{3} & \frac{1}{3} & 0 & 0 \\
0 & \frac{1}{3} & \frac{1}{3} & \frac{1}{3} & 0 \\
0 & 0 & \frac{1}{3} & \frac{1}{3} & \frac{1}{3}
\end{array}\right]
\end{aligned}
$$

Figure 1. Modification of the initial probability model.

\subsection{Retention mechanism of the optimal sample}

In the CE method, a sample is generated by sampling according to probability. Through iterations, the probability matrix is updated in which the values of some elements increase gradually and close to 1 . While, small probability events could still happen, which leads that the objective value of the optimal sample in an iteration is not always better than it in the last iteration. So the optimal sample in each iteration should be retained and added to the set of samples in the next iteration which can make the results of the iterations change monotonically and improve the quality of samples. In each layer of the Two-Layers CE algorithm, the optimal sample in each iteration is suitable to this retention mechanism.

\section{SIMULATION AND EXPERIMENT RESULTS}

Three different-scaled simulation instances are devised to test the effect and performance of the algorithm. Instance 1 has 5 machines 2 jobs (5 operations in total), Instance 2 has 6 machines 4 jobs (12 operations in total) and Instance 3 has 8 machines 8 jobs (43 operations in total). The TOU is defined as matrix $E P=\left[\begin{array}{c}7,9,12,14,18,23,24 \text { (h) } \\ 0.5,0.8,1.3,0.8,1.3,0.8,0.5 \text { (yuan) }\end{array}\right]$. Parameters of the Two-Layers CE algorithm are set as the follows: the first layer is to determine the machine assignment status, and the number of samples $N_{1}=n \times m$, quantile of elite samples $\rho_{1}=0.1$, learning rate $\alpha_{1}=0.8$; the second layer is to determine the operation sequence, and the number of samples $N_{2}=n \times \sqrt{n}$, quantile of elite samples $\rho_{2}=0.1$, learning rate $\alpha_{2}=0.8$. In order to avoid too few samples, if $N_{1}<50$ then $N_{1}=50$, the same 
to $N_{2}$. The algorithm is programmed by MATLAB R2013a, and carried out on a PC of Pentium(R) Dual-Core CPU, 2.6GHz and 2GB RAM.

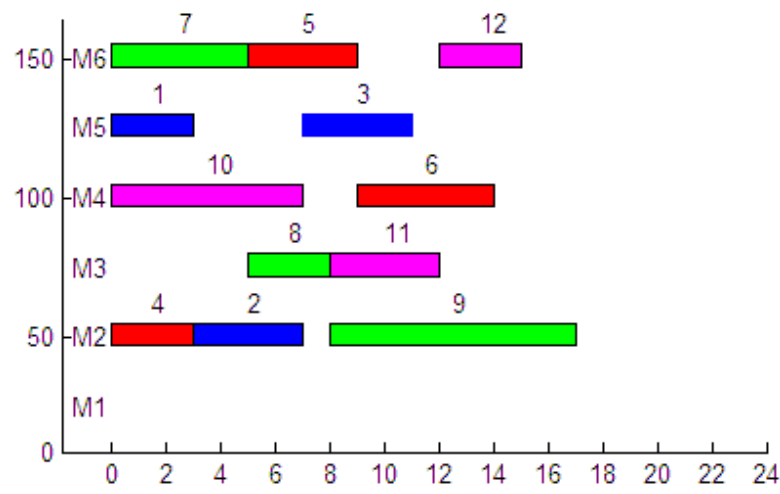

(a) $w=[1,0]$

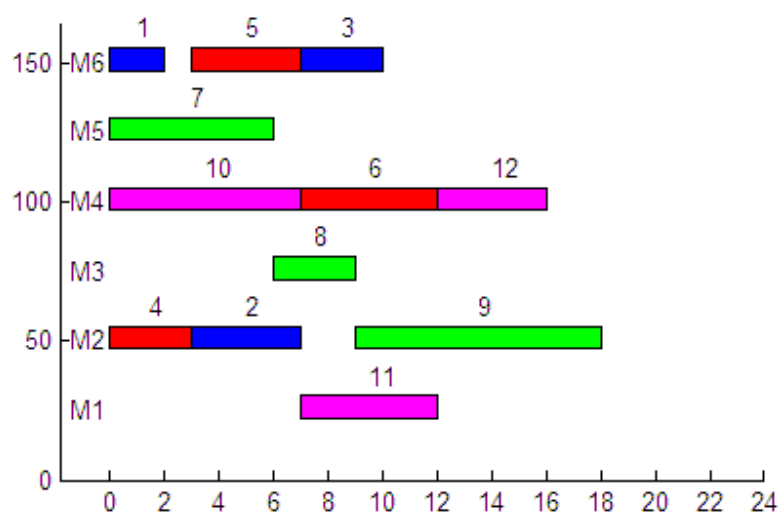

(b) $w=[0.6,0.4]$

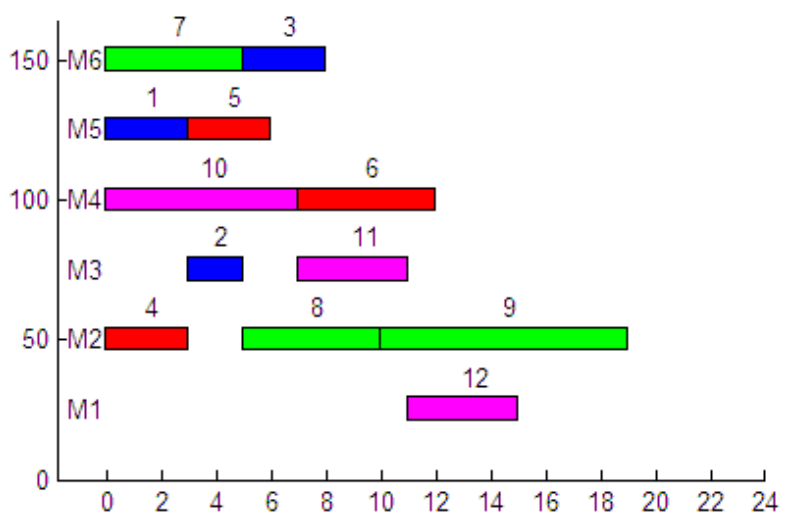

(c) $w=[0,1]$

Figure 2. Gantt charts for simulation result of Instance 2.

In order to analyze the influence to the two objectives of MS and EPC when they have different weight values in the total objective function, a weight vector is defined as $w=\left[w_{m s}, w_{e p c}\right]$. Here, $w_{m s}, w_{e p c} \in[0,1]$ and $w_{m s}+w_{e p c}=1$. Each instance is solved 15 times continuously with different values of $w$. Average results are shown in Table 2. It can be seen from Table 2, Instance 1 has small scale, short MS, so the TOU has small influence to it. That is, MS has little increase and EPC has little decrease with the increasing of $w_{\text {epc }}$; Instance 2 and Instance 3 have larger scales and longer MS, and they cover several electric price intervals, the TOU has larger influence to them. So, when the EPC is considered, MS has little increase but EPC decreases largely. In a word, no matter the scale of the problem, the TwoLayers CE algorithm can solve FJSP effectively and get the result of short MS with lower EPC.

Figure 2 shows the Gantt charts of Instance 2 with different values of $w$. We can see that with the objective of EPC considered, operations of jobs are arranged to process in valley or flat intervals of the TOU as far as possible so as to decrease the EPC. In addition, the idle time of machines is reduced also which can increase the efficiency of the machines.

\section{CONCLUSIONS}

The FJSP is studied and a Two-Layers CE algorithm is proposed to solve it. The objective is to find a reasonable schedule to minimize the MS and the EPC simultaneously. Based on the basic cross entropy method, we design the matrix encoding scheme and the probability model of the FJSP for each layer of the Two-Layers CE algorithm and modify the initial probability model according to some heuristic rules. Simulation experiments are implemented to verify the high performance of the algorithm.

\section{REFERENCES}

[1] Benziani, Y. \& Kacem, I. et al. 2014. Exact and heuristic methods for minimizing the total completion time in jobshops. Studies in Informatics and Control 23(1): 31-40.

[2] Blazewicz, J. \& Domschke, W. et al. 1996. The job shop scheduling problem: Conventional and new solution techniques. European Journal of Operational Research 93(1): 1-33.

[3] Blazewicz, J. \& Finke, G. et al. 1988. New trends in machine scheduling. European Journal of Operational Research 37:303-317.

[4] De Boer, P.T. \& Kroese, D.P. et al. 2005. A tutorial on the cross-entropy method. Annals of Operations Research 134(1): 19-67.

[5] Gao, L. \& Zhang, G.H. et al. 2012. Intelligent algorithm and its application for the flexible job-shop scheduling. Wuhan: HuaZhong University of Science \& Technology Press.

[6] Pezzella, F. \& Morganti, G. et al. 2008. A genetic algorithm for the flexible job-shop scheduling problem. Computers \& Operations Research 35(10): 3202-3212.

[7] Rubinstein, R.Y. 1999. The cross-entropy method for combinatorial and continuous optimization. Methodology and Computing in Applied Probability 1(2): 127-190.

[8] Zhang, G.H. \& Gao, L. et al. 2011. An effective genetic algorithm for the flexible job-shop scheduling problem. Expert Systems with Applications 38(4): 3563-3573. 